\title{
Evaluación de la resistencia al cizallamiento por compresión y falla en madera de uniones encoladas en maderas nacionales e importadas
}

(1) Quagliotti, S.

Contacto: squaglio@latu.org.uy

(1) Departamento de Proyectos Forestales, Laboratorio Tecnológico del Uruguay (LATU)

Recibido: 8/9/2008 - Aprobado: 29/11/2008

\section{Resumen}

En el presente trabajo se muestran los resultados de la evaluación de la resistencia al cizallamiento por compresión y falla en madera de uniones encoladas en Eucalyptus grandis, Pinus taeda, Pinus eliottii, lapacho y cedro.

El procedimiento se basó en las siguientes normas: ASTM D 5751-99, JIS K 6852-1994 y EN 205:1991. Las etapas más relevantes fueron las siguientes: encolado y corte de probetas, tratamientos de inmersión en agua a $30^{\circ} \mathrm{C}, 60^{\circ} \mathrm{C}, 100{ }^{\circ} \mathrm{C}$ y ensayo en máquina universal.

Se determinó la resistencia al cizallamiento por compresión, retención del poder adhesivo y porcentaje de falla en madera.

Es posible concluir que el PVA no es un adhesivo para ser utilizado en ambientes de alta humedad, debido a los bajos valores de resistencia y porcentaje de falla comparados con los valores en seco, como se muestra en las especies estudiadas luego de ser sometidas a los diferentes tratamientos.

Cabe resaltar que, una vez realizados los tratamientos de inmersión, las probetas encoladas con isocianato de E. grandis tienen los valores de retención del poder adhesivo más altos, como también menor disminución del porcentaje de falla en madera en comparación con las otras especies estudiadas.

Palabras clave: resistencia al cizallamiento, compresión, falla en madera, PVA, isocianato.

\section{$\underline{\text { Abstract }}$}

The results of the shear strength (by compression) and wood failure of bond unions in Eucalyptus grandis, Pinus taeda, Pinus eliottii, Tabebuia ipe y Cedrela spp are shown in the present work.

The process was based on the following standards: ASTM D 5751-99, JIS K 6852-1994 y EN 205:1991. The most relevant stages were bonding and the cutting of samples, water immersion treatment $\left(30,60,100{ }^{\circ} \mathrm{C}\right)$ and universal machine test.

Shear strength, Ratio wet/dry shear and Wood Failure percentage were determined.

As a conclusion, the PVA is not an adhesive to be used under high humidity conditions, due to the low values of shear strength and percentage of wood failure that the study species shown after water immersion treatment compared with the values under dry conditions.

Another relevant conclusion is that the samples of $\mathbf{E}$. grandis bonded with isocyanate shown the highest values of the Ratio wet/ dry shear after water immersion treatments, as well as a lower fall in the percentage of wood failure, compared with the other species under study.

Key Words: shear strength, compression, wood failure, PVA, isocyanate.

\section{Introducción}

La presente nota técnica es el complemento de la nota técnica $\mathrm{N}^{\mathrm{o}} 9$ (Evaluación de la resistencia a la adhesión y el porcentaje de falla en madera en Eucalyptus grandis, Pinus taeda, Pinus elliottii, Cedrela spp y Tabebuia Ipe). Estos estudios se vienen desarrollando dentro del Grupo de Encastres de Madera Sólida (GT6). Dicho grupo está integrado por la Asociación de Industriales de la Madera del Uruguay, la Universidad del Trabajo del Uruguay y el Laboratorio Tecnológico del Uruguay.

En el trabajo que aquí se presenta se analiza la adhesividad de cinco especies de madera: E. grandis, P. taeda, P. elliottii, cedro y lapacho.

La evaluación se realizó tomando en cuenta la resistencia al cizallamiento por compresión y el porcentaje de falla en madera.

\section{Materiales y Métodos}

Los materiales utilizados en el presente trabajo se detallan a continuación:

\section{MADERA}

- Eucalyptus grandis: se seleccionaron tablas de árboles de 12 años, sin poda y con raleo, provenientes del departamento de Rivera.

- Pinus taeda: se seleccionaron tablas de árboles de 24 años, con poda y raleo, provenientes del departamento de Río Negro.

- Pinus elliottii: se seleccionaron tablas de árboles de 24 años, con poda y raleo, provenientes del departamento de Río Negro y tablas de árboles de 40 años de trozas basales, sin poda y raleo provenientes del departamento de San José.

- cedro y lapacho: tablas de origen y edad desconocido suministradas por Bomel S.A (ADIMAU). 


\section{ADHESIVOS}

\section{Emulsión de Polivinil Acetato (PVA)}

Adhesivo líquido de naturaleza termoplástica pronto para uso, de color blanco a amarillo, de aplicación directa, prensado a temperatura ambiente. Alta resistencia en condiciones secas y baja resistencia en condiciones de alta humedad y temperatura.

Se utilizó PVA marca TORRENTE. Dicho adhesivo es una dispersión acuosa de Acetato de Polivinilo de alta viscosidad (120-200 ps), $\mathrm{pH}$ de $6-7$, el coloide protector es alcohol polivinílico, tamaño de partícula de 0,5 a 3 micrones. Se utilizaron dos emulsiones con diferente contenido no volátil de $49 \%$ (PVA A) y $44 \%$ (PVA B).

\section{Emulsión polímero - Isocianato}

Adhesivo de dos componentes, por un lado una resina que contiene un polímero acuoso y por otro un catalizador de isocianato. La resina y el catalizador deben mezclarse antes de aplicarse y la mezcla presenta un color blanco. Dicho adhesivo tiene alta resistencia a la humedad, al calor y a los solventes.

Se utilizó un Isocianato marca Koyo Bond KR-134, presenta una viscosidad de 100 ps a $25^{\circ} \mathrm{C}$, un contenido de sólidos de 60 $\%$ y un $\mathrm{pH}$ de 7,5 . La relación de mezcla entre resina y catalizador es de $100 / 15$ en peso.

Este estudio se basa en las siguientes normas:

- ASTM D 5751-99 (Adhesives Used for Laminate Joints in Nonstructural Lumber Products).

- JIS K 6852-1994 (Testing methods for shear strength of adhesive bonds by compression loading).

- EN 205:1991. Métodos de ensayo para adhesivos para la madera de uso no estructural.

A continuación se presenta la secuencia de etapas de realización:

\section{- Elaboración de listones para confeccionar probetas}

Para cada especie se elaboraron listones a partir de las tablas libres de defectos. Todos los listones poseen una sección transversal de corte radial. Las dimensiones de los mismos son: $300 \mathrm{~mm}$ de largo x $45 \mathrm{~mm}$ de ancho x $10 \mathrm{~mm}$ de espesor.

\section{- Determinación del contenido de humedad de los listones} De cada especie se seleccionaron 10 listones para determinar el contenido de humedad por método de secado en estufa. Primero se pesaron los listones y luego fueron colocados en estufa, secándose a $103 \pm 2{ }^{\circ} \mathrm{C}$ hasta llegar a peso constante.

Los valores de humedad se indican en la Tabla 1.

\begin{tabular}{|c|c|}
\hline Especie & $\begin{array}{c}\text { Contenido de } \\
\text { humedad (\%) }\end{array}$ \\
\hline Tabebuia ipe (lapacho) & 12,3 \\
\hline Cedrela spp. (cedro) & 13,7 \\
\hline P. taeda & 9,9 \\
\hline P. elliottii & 9,7 \\
\hline E. grandis & 10,1 \\
\hline
\end{tabular}

\section{- Determinación de la densidad de los listones}

A cada uno de los listones se les determinó el largo y el ancho utilizando un calibre digital, y el espesor utilizando un micrómetro digital. Luego se procedió al peso de los mismos, para el posterior cálculo de la densidad. Con los valores obtenidos se ranquearon los listones de menor a mayor valor de densidad, para poder encolar listones de similar densidad.

\section{- Acondicionamiento previo al encolado}

Los listones fueron acondicionados en una cámara climatizada a $20{ }^{\circ} \mathrm{C}$ y $65 \%$ de humedad relativa (H.R) hasta que alcanzaron el equilibrio luego de dos pesadas consecutivas de los testigos. El contenido de humedad al que llegaron los listones luego de ser acondicionados se muestra en la Tabla 2

\begin{tabular}{|l|c|}
\hline \multicolumn{1}{|c|}{ Especie } & $\begin{array}{c}\text { Contenido } \\
\text { de humedad (\%) }\end{array}$ \\
\hline Tabebuia ipe (lapacho) & 11,6 \\
\hline Cedrela spp. (cedro) & 13,2 \\
\hline P. taeda & 11,4 \\
\hline P. elliottii & 10,8 \\
\hline E. grandis & 10,5 \\
\hline
\end{tabular}

Tabla 2. Contenido de humedad de los listones acondicionados a 20 ${ }^{\circ} \mathrm{C}$ y $65 \%$ H.R según especie.

\section{- Encolado}

Una vez acondicionados, se encolaron pares de listones de similar densidad aplicando una carga de adhesivo de 250-300 g/ $\mathrm{m}^{2}$. Para el prensado se utilizó una prensa "SHINTO Compression Moulding Machine" (Figura 1) colocando los listones entre los platos de la prensa, y se utilizó una presión de 10 o $12 \mathrm{kgf} / \mathrm{cm}^{2}$ dependiendo de la especie. Para $E$. grandis y lapacho se utilizó 12 $\mathrm{kgf} / \mathrm{cm}^{2}$ y para los dos pinos y el cedro $10 \mathrm{kgf} / \mathrm{cm}^{2}$. El fraguado se realizó con las probetas sujetas a las prensas durante 24 horas, a temperatura y humedad ambiente.

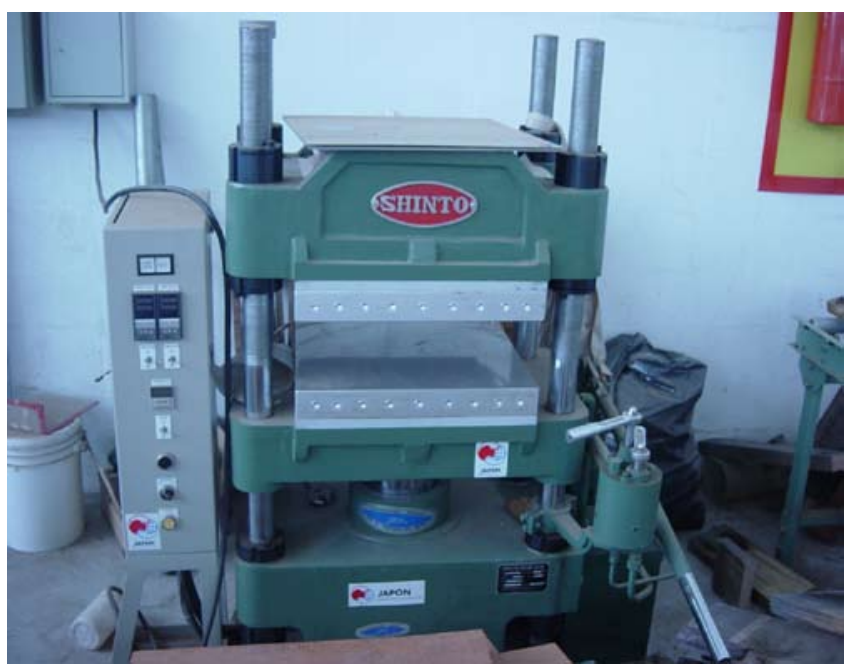

Figura 1. Prensa "SHINTO Compression Moulding Machine".

\section{- Corte de las probetas}

De cada par de listones encolados se obtuvieron 10 probetas escalonadas, como se muestra en la Figura 2. Cada probeta tiene un área de ensayo de $600 \mathrm{~mm}^{2}$ aproximadamente. 


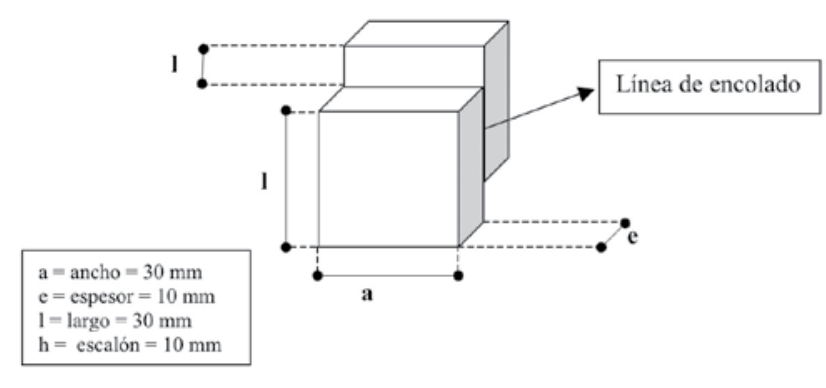

Figura 2. Geometría de las probetas escalonadas.

\section{- Acondicionamiento de las probetas previo al ensayo de compresión}

Se realizó un acondicionamiento a $20{ }^{\circ} \mathrm{C}$ y $65 \%$ de H.R (curado de las probetas) por un período de una semana.

\section{- Tratamientos realizados}

Los tratamientos a los que fueron sometidas las probetas pretenden crear condiciones de envejecimiento previas a la realización del ensayo. Además, se ensayaron probetas en condiciones secas, y antes de la realización de cada tratamiento se determinó el ancho y espesor de cada una en el área de ensayo.

\section{Tratamiento 1 (Condiciones secas)}

En este tratamiento las probetas luego de estar una semana en cámara climatizada a una H.R de $65 \%$ y $20{ }^{\circ} \mathrm{C}$ se ensayaron en condiciones secas.

\section{Tratamiento 2 (Inmersión en agua a $30^{\circ} \mathrm{C}$ )}

Las probetas son sumergidas en agua a $30{ }^{\circ} \mathrm{C}$ dentro de un baño termostatizado (Figura 3) con control de temperatura por un período de tres horas. Luego las probetas son colocadas debajo de agua a temperatura ambiente por 10 minutos, y a continuación son ensayadas en condiciones húmedas.

\section{Tratamiento 3 (Inmersión en agua a $60^{\circ} \mathrm{C}$ )}

Las probetas son sumergidas en agua a $60{ }^{\circ} \mathrm{C}$ dentro de un baño termostatizado con control de temperatura por un período de tres horas. Luego las probetas son colocadas en agua a temperatura ambiente por 10 minutos, y a continuación son ensayadas en condiciones húmedas.

\section{Tratamiento 4 (Inmersión en agua a $100{ }^{\circ} \mathrm{C}$ )}

Las probetas a ensayar en húmedo fueron sometidas a un tratamiento Húmedo-Seco-Húmedo, detallado a continuación:

Colocación de probetas en baño de agua a $100{ }^{\circ} \mathrm{C}$ durante cuatro horas.

Secado en estufa a $60{ }^{\circ} \mathrm{C}$ durante 20 horas.

Colocar probetas en agua fría por una hora.

Colocar probetas en baño de agua a $100{ }^{\circ} \mathrm{C}$ durante cuatro horas.

Este tratamiento solamente fue realizado en las probetas encoladas con Isocianato, debido a la agresividad del procedimiento. Para dicho tratamiento se utilizó un baño termostatizado con control de temperatura y una estufa de secado con circulación forzada.

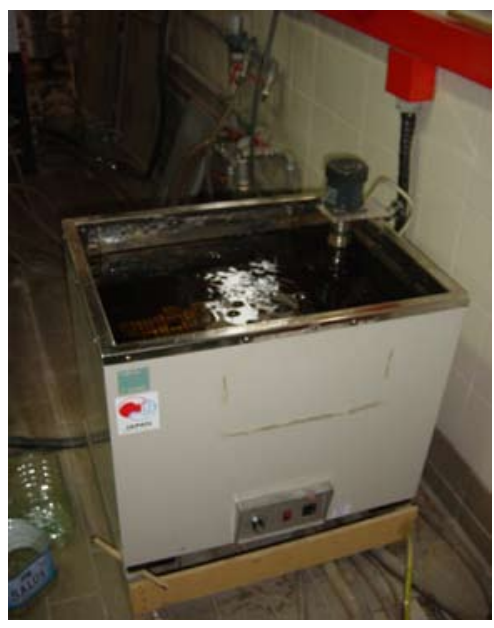

Figura 3. Baño termostatizado con control de temperatura.

\section{- Ensayo}

Se realizó en una máquina universal de ensayos dentro de una cámara climatizada a $20^{\circ} \mathrm{C}$ y $65 \%$ de humedad relativa, como se muestra en la Figura 4. Se aplicó compresión paralela a la unión encolada, con una velocidad de aplicación de $10 \mathrm{~mm} /$ minuto en todos los casos.

Se registró la carga máxima aplicada en Newton y se calculó la resistencia de la unión encolada como el esfuerzo unitario máximo, empleando la siguiente fórmula:

Resistencia al cizallamiento por compresión $\left(\mathrm{N} / \mathrm{mm}^{2}\right)=$ Carga máxima aplicada $(\mathrm{N}) /$ Área de ensayo $\left(\mathrm{mm}^{2}\right)$

Cabe aclarar que N/mm2 es igual a Megapascal (MPa).

Luego del ensayo se determinó mediante una inspección visual el porcentaje de falla en madera de cada probeta. Éste corresponde al cociente entre el área de ensayo donde se produjo la rotura en la madera y el área de ensayo de la probeta.

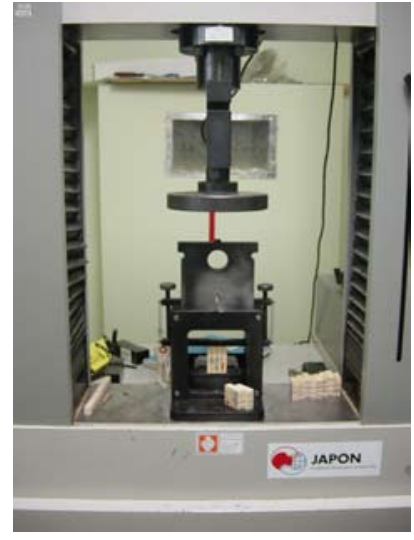

Figura 4. Máquina universal.

También se determinó la retención del poder adhesivo para los tres adhesivos, entendiéndose como tal el porcentaje entre los promedios de la resistencia al cizallamiento obtenido en las condiciones húmeda y seca respectivamente, o sea:

Retención del poder adhesivo $(\%)=\mathrm{H} / \mathrm{S} * 100$

Donde:

$\mathrm{H}=$ Promedio de la resistencia al cizallamiento según tratamiento húmedo

$\mathrm{S}=$ Promedio de la resistencia al cizallamiento en condiciones secas 


\section{Resultados}

\section{Resistencia al cizallamiento por compresión, retención del poder adhesivo y porcentaje de falla en madera}

En las siguientes tablas y gráficos se muestran los resultados obtenidos para cada especie, adhesivo y condición de ensayo. Para cada tratamiento se ensayaron 10 probetas por especie.

\section{PVA $49 \%$ de sólidos}

En las Tablas 3 y 4 se presentan los resultados de resistencia al cizallamientoporcompresión, retencióndelpoderadhesivoyelporcentaje de falla en madera para las probetas encoladas con PVA (mayor carga de sólidos) según tratamiento. Cabe aclarar que no fueron ensayadas probetas de cedro y lapacho, debido a que no se pudo conseguir madera suficiente de estas dos especies para poder confeccionar probetas.

\begin{tabular}{|c|c|c|c|c|c|c|c|c|c|}
\hline \multirow[b]{3}{*}{ Tratamiento } & \multicolumn{9}{|c|}{$\begin{array}{l}\text { Resistencia al cizallamiento } \\
\text { por compresión (MPa) y Retención } \\
\text { poder adhesivo }(\%)\end{array}$} \\
\hline & \multicolumn{3}{|c|}{ E. grandis } & \multicolumn{3}{|c|}{ P. taeda } & \multicolumn{3}{|c|}{ P. elliottii } \\
\hline & \multicolumn{2}{|c|}{ MPa } & $\begin{array}{l}\mathrm{H} / \mathrm{S} \\
(\%)\end{array}$ & & $\mathrm{MPa}$ & $\begin{array}{l}\mathrm{H} / \mathrm{S} \\
(\%)\end{array}$ & \multicolumn{2}{|c|}{ MPa } & $\begin{array}{l}\mathrm{H} / \mathrm{S} \\
(\%)\end{array}$ \\
\hline \multirow{2}{*}{$\begin{array}{l}\text { Tratamiento } 1 . \\
\text { Condiciones } \\
\text { secas }\end{array}$} & $X$ & 10,5 & \multirow[b]{2}{*}{---- } & $\mathrm{X}$ & 11,6 & \multirow[b]{2}{*}{---- } & $\mathrm{X}$ & 10,0 & \multirow[b]{2}{*}{---- } \\
\hline & $\mathrm{S}$ & 0,54 & & $\mathrm{~S}$ & 1,22 & & $\mathrm{~S}$ & 1,0 & \\
\hline \multirow{2}{*}{$\begin{array}{l}\text { Tratamiento } 2 \text {. } \\
\text { Inmersión } \\
\text { agua } 30^{\circ} \mathrm{C}\end{array}$} & $\mathrm{X}$ & 4,1 & \multirow[b]{2}{*}{39} & $\mathrm{X}$ & 0,8 & \multirow[b]{2}{*}{7} & $\mathrm{X}$ & 0,3 & \multirow[b]{2}{*}{3} \\
\hline & S & 0,34 & & $S$ & 0,32 & & $\mathrm{~S}$ & 0,12 & \\
\hline \multirow{2}{*}{$\begin{array}{l}\text { Tratamiento } 3 \text {. } \\
\text { Inmersión en } \\
\text { agua } 60^{\circ} \mathrm{C}\end{array}$} & $\mathrm{X}$ & 2,0 & \multirow[b]{2}{*}{19} & $\mathrm{X}$ & 0,2 & \multirow[b]{2}{*}{2} & $\mathrm{X}$ & 0,1 & \multirow[b]{2}{*}{1} \\
\hline & $\mathrm{S}$ & 0,65 & & S & 0,07 & & $\mathrm{~S}$ & 0,08 & \\
\hline
\end{tabular}

Tabla 3. Resistencia al cizallamiento por compresión, retención del poder adhesivo para cada especie y tratamiento utilizando probetas encoladas con PVA A (49 \% sólidos).

\begin{tabular}{|c|c|c|c|}
\hline & \multicolumn{3}{|c|}{ Falla en Madera (\%) } \\
\hline Tratamiento & E. grandis & P. taeda & P. elliottii \\
\hline $\begin{array}{c}\text { Tratamiento 1. } \\
\text { Condiciones secas }\end{array}$ & 87 & 64 & 66 \\
\hline $\begin{array}{c}\text { Tratamiento } 2 . \\
\text { Inmersión en agua } 30^{\circ} \mathrm{C}\end{array}$ & 12 & 0 & 0 \\
\hline $\begin{array}{c}\text { Tratamiento } 3 . \\
\text { Inmersión en agua } 60^{\circ} \mathrm{C} .\end{array}$ & 1 & 0 & 4 \\
\hline
\end{tabular}

Tabla 4. Falla en madera para cada especie y tratamiento utilizando probetas encoladas con PVA A ( $49 \%$ sólidos).

Los datos de las Tablas 3 y 4 se representan gráficamente en los Gráficos 1 y 2.

\section{$\square$ Condiciones secas $\square$ Inmersión $30^{\circ} \mathrm{C} \quad \square$ Inmersión $60^{\circ} \mathrm{C}$}

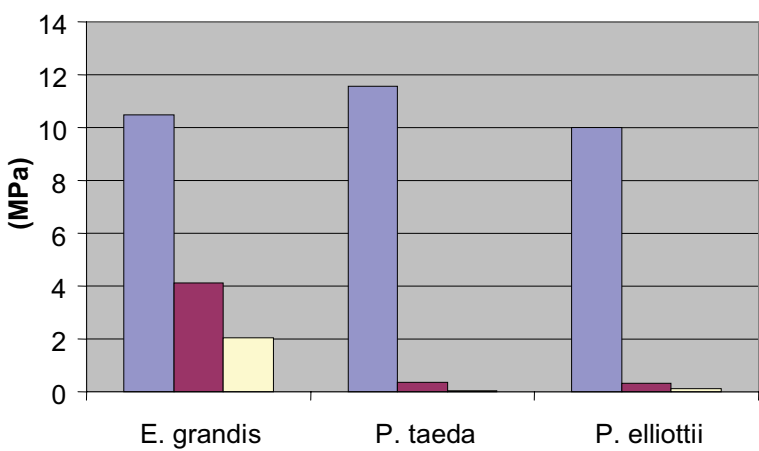

Gráfico 1. Resistencia al cizallamiento por compresión según tratamiento para probetas encoladas con PVA $49 \%$ de sólidos.

\section{$\square$ Condiciones secas $\quad \square$ Inmersión $30^{\circ} \mathrm{C} \quad \square$ Inmersión $60^{\circ} \mathrm{C}$}

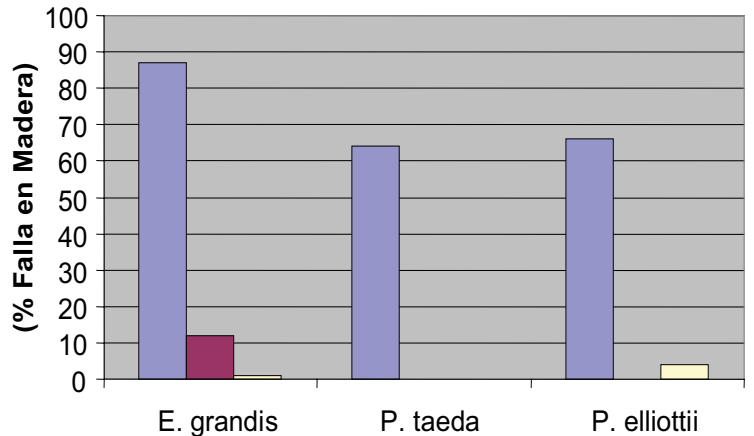

Gráfico 2. Falla en madera según tratamiento para probetas encoladas con encolados con PVA $49 \%$ de sólidos.

Se observa que los valores de resistencia en condiciones secas son similares para las tres especies estudiadas, sin embargo E. grandis presenta valores superiores de porcentaje de falla en madera (87\%) comparado con los pinos (64 \% y $66 \%$ ).

Como era esperado, luego de los tratamientos en inmersión en agua a $30^{\circ} \mathrm{C}$ y $60{ }^{\circ} \mathrm{C}$ los valores de resistencia y falla en madera disminuyen en todos los casos. Para ambos tipos de pino, luego de los tratamientos los valores están cercanos a cero, tanto para la resistencia como para la falla en madera.

Asimismo, la retención del poder adhesivo es baja para las tres especies y resulta mínima en las dos especies de pinos donde es menor al $10 \%$ después de los dos tratamientos. Esto se debe a que el PVA, adhesivo hidrosoluble, es poco resistente a condiciones de alta humedad.

\section{PVA 44 \% de Sólidos}

En las Tablas 5 y 6 se presentan los resultados de resistencia al cizallamiento por compresión, retención del poder adhesivo y porcentaje de falla en madera para las probetas encoladas con PVA (menor carga de sólidos) según tratamiento.

Para E. grandis, $P$. taeda y P. elliottii, luego de los tratamientos de inmersión en agua la resistencia disminuye considerablemente en todas las especies, no registrándose valores de resistencia para $P$. taeda luego de la inmersión a $30^{\circ} \mathrm{C}$ y para $P$. elliottii luego de ambos tratamientos.

El porcentaje de falla en madera desciende en las cinco especies después de los tratamientos, registrándose valores menores al $5 \%$ de falla en todas ellas. 


\begin{tabular}{|c|c|c|c|c|c|c|c|c|c|c|c|c|c|c|c|}
\hline \multirow{3}{*}{ Tratamiento } & \multicolumn{15}{|c|}{ Resistencia al cizallamiento por compresión (MPa) y retención poder adhesivo (\%) } \\
\hline & \multicolumn{3}{|c|}{ E. grandis } & \multicolumn{3}{|c|}{ Cedrela spp (cedro) } & \multicolumn{3}{|c|}{$\begin{array}{c}\text { Tabebuia ipe } \\
\text { (lapacho) }\end{array}$} & \multicolumn{3}{|c|}{ P. taeda } & \multicolumn{3}{|c|}{ P. elliottii } \\
\hline & \multicolumn{2}{|c|}{ MPa } & \multirow{3}{*}{$\begin{array}{c}\mathrm{H} / \mathrm{S}(\%) \\
---\end{array}$} & \multicolumn{2}{|c|}{ MPa } & \multirow{3}{*}{$\begin{array}{l}\mathbf{H} / \mathbf{S} \\
\mathbf{( \% )} \\
\end{array}$} & \multicolumn{2}{|c|}{ MPa } & \multirow{3}{*}{$\begin{array}{l}\mathbf{H} / \mathbf{S} \\
(\%) \\
---\end{array}$} & \multicolumn{2}{|c|}{ MPa } & \multirow{3}{*}{$\begin{array}{c}\mathrm{H} / \mathrm{S}(\%) \\
---\end{array}$} & \multicolumn{2}{|c|}{ MPa } & \multirow{3}{*}{$\begin{array}{c}\text { H/S (\%) } \\
---\end{array}$} \\
\hline \multirow{2}{*}{$\begin{array}{c}\text { Tratamiento } 1 . \\
\text { Condiciones secas }\end{array}$} & $\mathbf{X}$ & 9,6 & & $\mathbf{X}$ & 9,2 & & $\mathbf{X}$ & 8,2 & & $\mathbf{X}$ & 11,3 & & $\mathbf{X}$ & 8,7 & \\
\hline & $\mathrm{S}$ & 0,92 & & $\mathrm{~S}$ & 1,4 & & $\mathrm{~S}$ & 1,7 & & $\mathrm{~S}$ & 1,93 & & $\mathrm{~S}$ & 1,1 & \\
\hline \multirow{2}{*}{$\begin{array}{l}\text { Tratamiento } 2 . \\
\text { Inmersión en agua } \\
30^{\circ} \mathrm{C}\end{array}$} & $\mathbf{X}$ & 3,7 & \multirow[t]{2}{*}{39} & $\mathbf{X}$ & 2,1 & \multirow[t]{2}{*}{23} & $\mathbf{X}$ & 0,6 & \multirow[t]{2}{*}{7} & $\mathbf{X}$ & $\mathbf{0 , 4}$ & \multirow[t]{2}{*}{4} & $\mathbf{X}$ & $\mathbf{0 , 1}$ & \multirow[t]{2}{*}{1} \\
\hline & $\mathrm{S}$ & 0,91 & & $\mathrm{~S}$ & 0,93 & & $\mathrm{~S}$ & 0,34 & & $\mathrm{~S}$ & 0,17 & & $\mathrm{~S}$ & 0,11 & \\
\hline \multirow{2}{*}{$\begin{array}{l}\text { Tratamiento } 3 . \\
\text { Inmersión en agua } \\
60^{\circ} \mathrm{C}\end{array}$} & $\mathbf{X}$ & 1,0 & \multirow[t]{2}{*}{10} & $\mathbf{X}$ & $\mathbf{0 , 9}$ & \multirow{2}{*}{10} & $\mathbf{X}$ & 0,2 & \multirow[t]{2}{*}{2} & $\mathbf{X}$ & $\mathbf{0 , 0}$ & \multirow[t]{2}{*}{0} & $\mathbf{X}$ & 0 & \multirow{2}{*}{0} \\
\hline & $\mathrm{S}$ & 0,71 & & $\mathrm{~S}$ & 0,51 & & $\mathrm{~S}$ & 0,21 & & $\mathrm{~S}$ & 0,0 & & $\mathrm{~S}$ & 0 & \\
\hline
\end{tabular}

Tabla 5. Resistencia al cizallamiento por compresión, retención del poder adhesivo para cada especie y tratamiento utilizando probetas encoladas con PVA B (44 \% sólidos)

\begin{tabular}{|c|c|c|c|c|c|}
\hline & \multicolumn{5}{|c|}{ Falla en madera (\%) } \\
\hline Tratamiento & 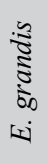 & 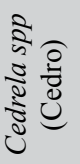 & 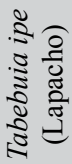 & 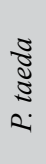 & 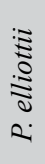 \\
\hline $\begin{array}{c}\text { Tratamiento } 1 . \\
\text { Condiciones secas }\end{array}$ & 78 & 82 & 29 & 47 & 42 \\
\hline $\begin{array}{c}\text { Tratamiento } 2 . \\
\text { Inmersión en agua } \\
30^{\circ} \mathrm{C} \\
\end{array}$ & 4 & 4 & 4 & $\mathbf{0}$ & 1 \\
\hline $\begin{array}{c}\text { Tratamiento } 3 . \\
\text { Inmersión en agua } \\
60^{\circ} \mathrm{C} \\
\end{array}$ & 4 & 5 & 3 & $\mathbf{0}$ & $\mathbf{0}$ \\
\hline
\end{tabular}

Tabla 6. Falla en madera para cada especie y tratamiento, utilizando probetas encoladas con PVA B (44 \% sólidos).

Los datos de las Tablas 5 y 6 se representan gráficamente en los Gráficos 3 y 4.

Al comparar los resultados en condiciones secas, se observa que P. taeda presenta los valores más altos de resistencia con 11,3 $\mathrm{MPa}$ y que las demás especies presentan valores dentro de un rango de 8 a $10 \mathrm{MPa}$.

La diferencia mayor se encuentra en el porcentaje de falla en madera, donde $E$. grandis y cedro presentan valores cercanos al 80 $\%$, en comparación con las otras tres especies que no superan el 50 $\%$ de falla.

\section{$\square$ Condiciones secas $\square$ Inmersión $30^{\circ} \mathrm{C} \quad \square$ Inmersión $60^{\circ} \mathrm{C}$}

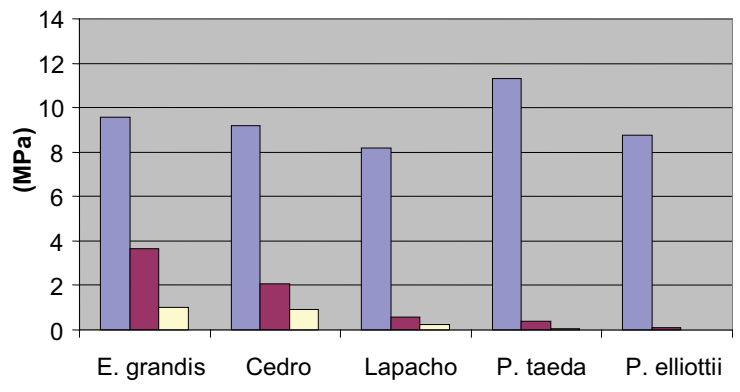

Gráfico 3. Resistencia al cizallamiento por compresión según tratamiento para probetas encoladas con PVA $44 \%$ sólidos

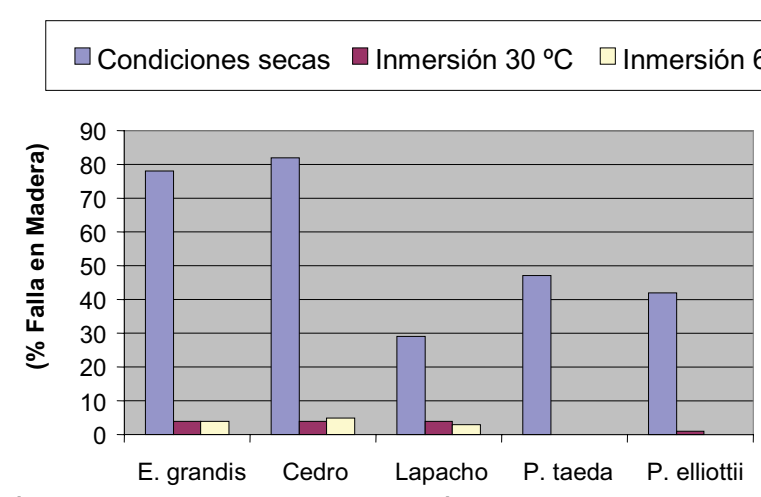

Gráfico 4. Falla en madera según tratamiento para probetas encoladas con PVA $44 \%$ sólidos

En relación a la retención del poder adhesivo se produce un comportamiento similar que con el PVA de mayor carga de sólidos. Esto se debe a que al absorber la madera y el adhesivo humedad del ambiente, cambian las características de la unión encolada y, por tanto, cuanto más severo es el tratamiento menor es la retención del poder adhesivo.

Esto se explica porque algunas de las principales causas del desencolado de adhesivos de Acetato de Polivinilo (PVA) son e ambiente excesivamente húmedo y la falta de resistencia al agua o a la humedad del adhesivo.

Comparando las probetas encoladas con ambos tipos de PVA y ensayadas en condiciones secas, se observa que a mayor carga de sólidos los valores de resistencia al cizallamiento y porcentaje de falla en madera son mayores.

Esto resulta más notorio en el porcentaje de falla que en la resistencia, y es aún más evidente la diferencia para las probetas de los dos tipos de pino, donde las encoladas con el PVA de mayor carga de sólidos tienen un valor de falla en madera promedio de $65 \%$, y las encoladas con el PVA de menor carga de sólidos, de $45 \%$. Esto se puede ver conjuntamente en las Tablas 3, 4, 5 y 6.

\section{Isocianato}

En las Tablas 7 y 8 se observan los resultados de la resistencia al cizallamiento por compresión, retención del poder adhesivo y el porcentaje de falla en madera para las distintas especies y tratamientos utilizando probetas encoladas con isocianato.

Se observa claramente que las probetas de lapacho presentan los valores más altos de resistencia, salvo para el tratamiento de inmersión 


\begin{tabular}{|c|c|c|c|c|c|c|c|c|c|c|c|c|c|c|c|}
\hline \multirow{3}{*}{ Tratamiento } & \multicolumn{15}{|c|}{ Resistencia al cizallamiento por compresión (MPa) y retención poder adhesivo (\%) } \\
\hline & \multicolumn{3}{|c|}{ E. grandis } & \multicolumn{3}{|c|}{$\begin{array}{l}\text { Cedrela spp } \\
\text { (cedro) }\end{array}$} & \multicolumn{3}{|c|}{$\begin{array}{c}\text { Tabebuia ipe } \\
\text { (lapacho) }\end{array}$} & \multicolumn{3}{|c|}{ P. taeda } & \multicolumn{3}{|c|}{ P. elliottii } \\
\hline & \multicolumn{2}{|c|}{ MPa } & $\begin{array}{l}\mathrm{H} / \mathrm{S} \\
(\%)\end{array}$ & \multicolumn{2}{|c|}{ MPa } & $\begin{array}{l}\mathbf{H} / \mathrm{S} \\
(\%)\end{array}$ & \multicolumn{2}{|c|}{ MPa } & $\begin{array}{l}\mathbf{H} / \mathbf{S} \\
(\%)\end{array}$ & \multicolumn{2}{|c|}{ MPa } & $\begin{array}{l}\mathrm{H} / \mathrm{S} \\
(\%)\end{array}$ & \multicolumn{2}{|c|}{ MPa } & $\begin{array}{l}\mathbf{H} / \mathrm{S} \\
(\%)\end{array}$ \\
\hline \multirow{2}{*}{ Tratamiento 1. Condiciones secas } & $\mathrm{X}$ & 10,1 & & $\mathrm{X}$ & 12,1 & \multirow{2}{*}{----} & $\mathrm{X}$ & 17,9 & \multirow{2}{*}{----} & $\mathrm{X}$ & 12,1 & \multirow{2}{*}{----} & $\mathrm{X}$ & 9,5 & \multirow{2}{*}{---} \\
\hline & $\mathrm{S}$ & 1,1 & & $\mathrm{~S}$ & 1,5 & & $\mathrm{~S}$ & 3,3 & & $\mathrm{~S}$ & 1,1 & & $\mathrm{~S}$ & 2,2 & \\
\hline \multirow{2}{*}{$\begin{array}{l}\text { Tratamiento } 2 . \\
\text { Inmersión agua } 30^{\circ} \mathrm{C}\end{array}$} & $X$ & 5,8 & \multirow[t]{2}{*}{91} & $X$ & 9,0 & \multirow[t]{2}{*}{74} & $X$ & 14,8 & \multirow[t]{2}{*}{83} & $\mathrm{X}$ & 5,7 & \multirow[t]{2}{*}{47} & $X$ & 4,3 & \multirow[t]{2}{*}{45} \\
\hline & $\mathrm{S}$ & 0,58 & & S & 2,0 & & $\bar{S}$ & 2,7 & & $\bar{S}$ & 0,58 & & $\mathrm{~S}$ & 1,0 & \\
\hline \multirow{2}{*}{$\begin{array}{l}\text { Tratamiento } 3 \text {. } \\
\text { Inmersión en agua } \quad 60^{\circ} \mathrm{C}\end{array}$} & $\mathrm{X}$ & 4,9 & \multirow{2}{*}{68} & $\mathrm{X}$ & 6,0 & \multirow{2}{*}{50} & $\mathrm{X}$ & 9,8 & \multirow{2}{*}{55} & $\mathrm{X}$ & 4,9 & \multirow{2}{*}{40} & $X$ & 4,1 & \multirow{2}{*}{43} \\
\hline & $\mathrm{S}$ & 0,55 & & $\bar{S}$ & 1,7 & & $\bar{S}$ & 4,1 & & $\bar{S}$ & 0,55 & & $\mathrm{~S}$ & 0,64 & \\
\hline \multirow{2}{*}{$\begin{array}{l}\text { Tratamiento } 4 . \\
\text { Inmersión en agua a } 100^{\circ} \mathrm{C}\end{array}$} & $x$ & 4,3 & \multirow{2}{*}{72} & $X$ & 2,8 & \multirow[t]{2}{*}{23} & $X$ & 4,6 & \multirow[t]{2}{*}{26} & $X$ & 4,3 & \multirow[t]{2}{*}{36} & $X$ & 3,3 & \multirow[t]{2}{*}{35} \\
\hline & $S$ & 1,5 & & $S$ & 1,3 & & $S$ & 2,4 & & $S$ & 1,5 & & $S$ & 1,5 & \\
\hline
\end{tabular}

Tabla 7. Resistencia al cizallamiento por compresión, retención del poder adhesivo para cada especie y tratamiento utilizando probetas encoladas con Isocianato.

en agua a $100{ }^{\circ} \mathrm{C}$, aunque es la especie que presenta valores de falla en madera más bajos luego de todos los tratamientos. Los elevados valores de resistencia están dados por la alta densidad que presenta el lapacho y no por una unión encolada resistente, lo cual se explica por el bajo porcentaje de falla en madera. El mismo se debe a que en especies más densas existe menor volumen de espacio libre que permita la entrada del adhesivo, no facilitando una buena adhesión mecánica.

\begin{tabular}{|c|c|c|c|c|c|}
\hline & \multicolumn{5}{|c|}{ Falla en madera (\%) } \\
\hline Tratamiento & 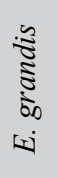 & 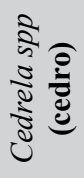 & 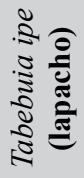 & 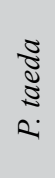 & 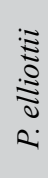 \\
\hline $\begin{array}{c}\text { Tratamiento } 1 . \\
\text { Condiciones secas. }\end{array}$ & 85 & 89 & 46 & 76 & 78 \\
\hline $\begin{array}{c}\text { Tratamiento } 2 . \\
\text { Inmersión agua } \\
30^{\circ} \mathrm{C} .\end{array}$ & 85 & 59 & 26 & 76 & 43 \\
\hline $\begin{array}{c}\text { Tratamiento } 3 . \\
\text { Inmersión en agua } \\
60^{\circ} \mathrm{C} .\end{array}$ & 85 & 53 & 32 & 74 & 55 \\
\hline $\begin{array}{c}\text { Tratamiento } 4 . \\
\text { Inmersión en agua } \\
100^{\circ} \mathrm{C} .\end{array}$ & 74 & 17 & 13 & 49 & 25 \\
\hline
\end{tabular}

Tabla 8. Falla en madera para cada especie y tratamiento utilizando probetas encoladas con Isocianato.

Los datos de las Tablas 7 y 8 se representan gráficamente en los Gráficos 5 y 6 .

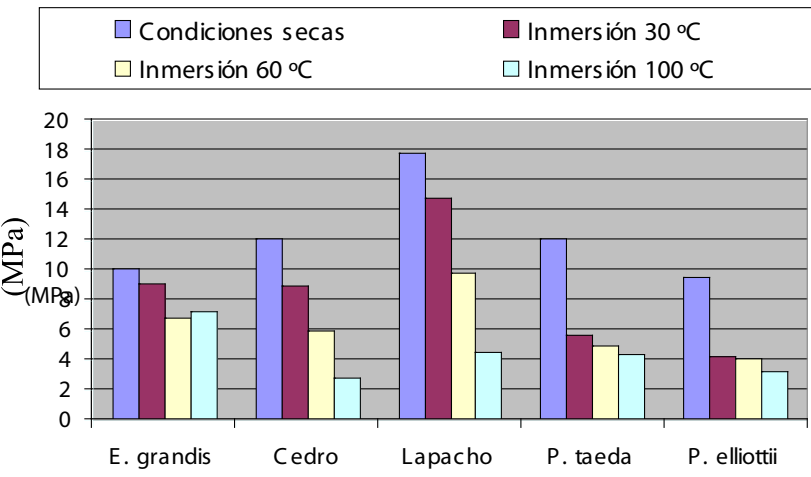

Gráfico 5. Resistencia al cizallamiento por compresión según tratamiento para probetas encoladas con Isocianato.

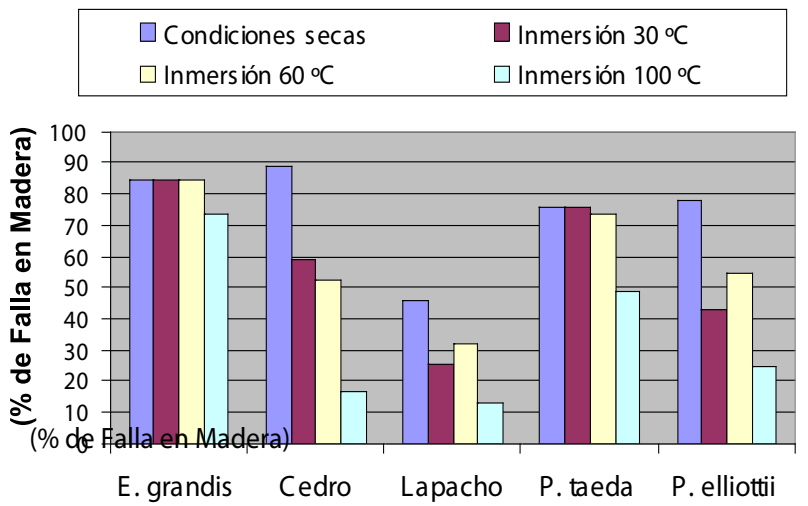

Gráfico 6. Falla en madera según tratamiento para probetas encoladas con Isocianato.

Efectuada la comparación de probetas de E. grandis, cedro, $P$. taeda y $P$. Elliottii, las de cedro son las que presentan los valores más altos de resistencia y porcentaje de falla, $12,1 \mathrm{MPa}$ y $89 \%$, respectivamente. El lapacho no fue considerado en esta comparación ya que sus valores de resistencia están dados en mayor medida por la densidad de la especie que por la unión encolada en sí.

$E$. grandis es la especie que presenta los mayores resultados 
de falla en madera luego de los tratamientos de inmersión en agua. Mantiene el mismo valor de $85 \%$ de falla luego de las inmersiones en agua a $30^{\circ} \mathrm{C}$ y $60{ }^{\circ} \mathrm{C}$, para descender a un valor de $74 \%$ luego del tratamiento de inmersión a $100^{\circ} \mathrm{C}$. La retención del poder adhesivo es la más alta de todas las especies estudiadas luego de los tratamientos.

Este resultado describe una especie que presenta una buena unión encolada, con alta resistencia tanto en seco como en condiciones húmedas, y un porcentaje de falla en madera alto en todos los casos, lo que indica una buena penetración del adhesivo para formar una unión fuerte.

\section{Conclusiones}

Utilizando PVA como adhesivo en condiciones secas, a mayor carga de sólidos mayor es la resistencia al cizallamiento y mayor es el porcentaje de falla en madera. Es posible visualizar esta relación en las dos especies de pino estudiadas (Tablas 3, 4, 5 y 6 y Gráficos 1 al 4).

PVA no es un adhesivo para ser utilizado en ambientes de alta humedad, observándose un debilitamiento de la unión con respecto al ensayo en condiciones secas en todos los casos, ya que luego de los tratamientos de inmersión en agua los valores obtenidos de resistencia y porcentaje de falla disminuyen.

Las dos especies que presentan mejor adhesividad en condiciones secas usando PVA como adhesivo son E. grandis y cedro. Si bien no existen mayores diferencias en los valores de resistencia con respecto a los pinos y al lapacho, presentan un mejor resultado en el porcentaje de falla en madera, lo que implica una mejor unión encolada (Tablas 5 y 6$)$.

Utilizando Isocianato como adhesivo, después de ensayar probetas expuestas a los diferentes tratamientos, se observa en general que a medida que aumenta la rigurosidad de las condiciones del tratamiento, la unión encolada se ve debilitada y tanto la resistencia al cizallamiento como el porcentaje de falla en madera disminuyen para todas las especies estudiadas.

Las dos especies que presentan mejor adhesividad utilizando Isocianato como adhesivo en condiciones secas son $E$. grandis y cedro: ambas presentan los valores más altos de resistencia y falla en madera.

En condiciones húmedas la especie que se comparta mejor es $E$. grandis, dado que las probetas encoladas con Isocianato presentan los valores de retención del poder adhesivo más altos luego de realizar los tratamientos de inmersión, y es la especie en la que, comparativamente, disminuye menos el porcentaje de falla en madera. Se concluye que frente al ataque del agua en los tratamientos, la unión $E$. grandisIsocianato, es la que se degrada menos, por lo que es posible concluir que se trata de la mejor unión relevada.

Las probetas de P. taeda presentan mayor proporción de madera de leño tardío respecto al leño temprano, lo cual hace que su densidad sea más alta. Por ende también presentan valores de resistencia al cizallamiento más altos que lo esperado. Esto se debe a la resistencia de la madera, como en el caso del lapacho, y no por la resistencia de la unión encolada, que presenta valores bajos de porcentaje de falla en madera.
- JAPANESE STANDARD ASSOCIATION (Japan). JIS K 6852. Testing methods for shear strength of adhesive bonds by compression loading. Tokio: JSA, 1994.

- PÉREZ DEL CASTILlO, A.; BENITES Maciel, L. Propiedades mecánicas y resistencia de uniones encoladas de vigas laminadas. Proyecto de tecnología de ensayo de productos forestales, LATUJICA (1998-2003). Montevideo: LATU, 2002. (Informe de Investigación. Serie Forestales; 10)

- QUAGLIOTTI ESTRADÉ, S. Evaluación de la resistencia a la adhesión y el porcentaje de falla en madera en Eucalyptus grandis, Pinus taeda, Pinus elliottii, Cedrela spp y Tabebuia ipe. Montevideo: LATU, 2007. (Nota Técnica; 9).

\section{Referencias}

- ASTM INTERNATIONAL (United States). D5751: Adhesives used for laminate joints in nonstructural lumber products. West Conshohocken: ASTM, 1999.

- BENITES MACIEL, L. Adhesividad de maderas nacionales. Proyecto de tecnología de ensayo de productos forestales, LATUJICA (1998-2003). Montevideo: LATU, 2003. (Informe de Investigación. Serie Forestales; 12)

- COMITÉ EUROPEO DE NORMALIZACIÓN (Bélgica). EN 205: Métodos de ensayo para adhesivos para la madera de uso no estructural. Bruselas: CEN, 1991. 